\title{
Efficacy of Unilateral Stenting in Idiopathic Intracranial Hypertension with Bilateral Venous Sinus Stenosis
}

\author{
A Case Report

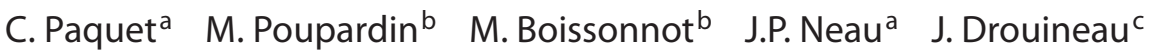 \\ Departments of a Neurology, ${ }^{\mathrm{b}}$ Ophthalmology, and ${ }^{\mathrm{c}}$ Radiology, CHU, Poitiers, France
}

Dear Sir,

As the pathogenesis of idiopathic intracranial hypertension (IIH) remains elusive, different medical and surgical treatments merit consideration.

\section{Case Report}

A 60-year-old woman was admitted for headaches and reduced visual acuity $(6 / 10$ right, 5/10 left). She presented bilaterally increased papilla volumes and bilateral deficits in the inferior quadrant. Brain MRI showed fluid accumulation in the optic nerve sheaths, reduced ventricle size and a normal sella. CSF pressure was 30 $\mathrm{cm}$ of water. The diagnosis of IIH was made. The removal of $40 \mathrm{ml}$ of CSF failed to alleviate symptoms. Acetazolamide treatment was initiated but, as a result of major side effects, was terminated prematurely by the patient. Subsequently, she refused further medical treatment. Symptoms worsened and the patient was readmitted. CSF pressure was $38 \mathrm{~cm}$ of water. A brain venous angiogram was performed, revealing bilateral stenosis of the transverse sinuses. The lead catheter could not pass through the stenosis on the left side. On the right side, the absolute venous pressure was $33 \mathrm{~mm}$ above and $18 \mathrm{~mm}$ below, and a stent was inserted by endovascular technique (fig. 1). The patient was hepa- rinized during the procedure, followed by long-term therapy with clopidogrel. Clinical improvement was immediate, marked by alleviation of headaches, regression of the papillary edema, and a return to normal visual acuity (10/10 right, 9/10 left). In this case angioplastic intervention proved efficacious, and no other surgical or medical treatment was performed.
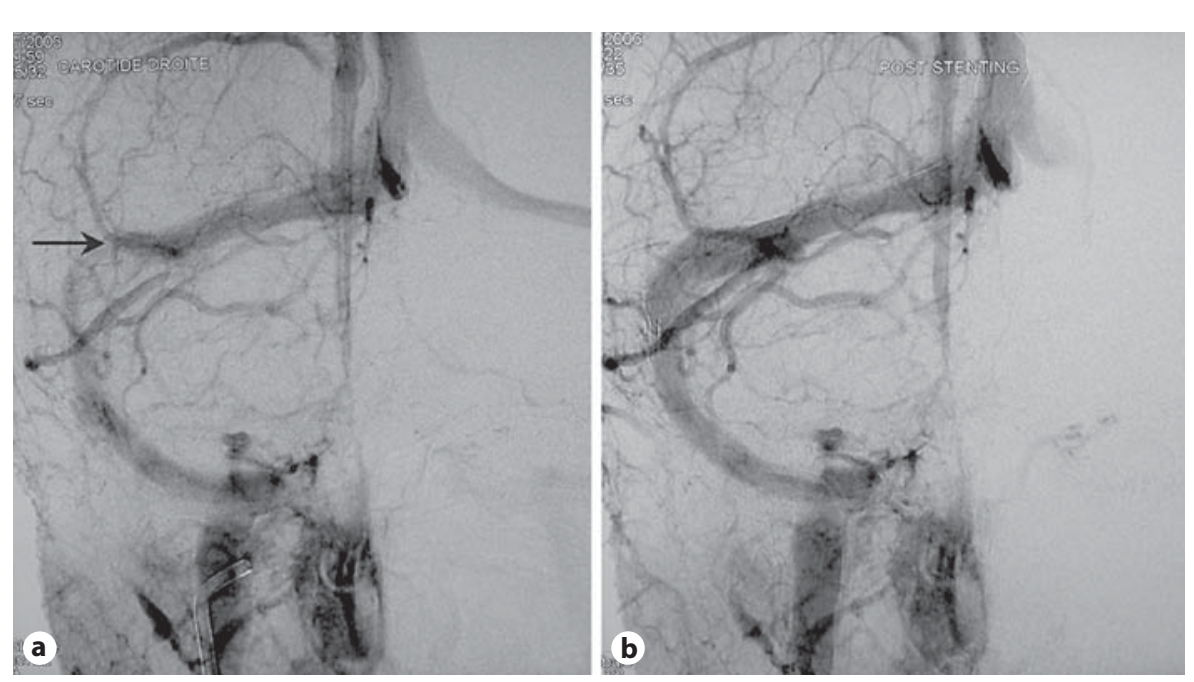

Fig. 1. a Stenosis of the transverse sinus before stenting. b Transverse sinus after stenting.

\section{Discussion}

Two hypotheses are proposed for the pathophysiology of IIH, and it is difficult to clearly define a suitable therapeutic target which can halt or prevent visual loss. The first hypothesis is an imbalance between CSF production and resorption. Medical and surgical interventions tend to reduce 
intracranial pressure, but comparative, randomized, prospective studies are lacking. The carbon anhydrase inhibitors acetazolamide and topiramate can efficiently diminish CSF production. Lumbar punctures directly reduce intracranial pressure and are essential in the immediate treatment of acute visual loss. The risk of low pressure headaches and the unpopularity of repeated lumbar punctures have led to a phaseout of this procedure. CSF ventricular shunts and decompression of the optic nerves do not seem to be very efficient and may present evident risks $[1,2]$.

The second hypothesis is linked to venous obstacles. Higgins et al. [3] reported a group of 12 patients with stenosis of the transverse sinus and various grades of venous pressure. No patient showed deterio- ration after angioplastic treatment, while it proved beneficial in more than $50 \%$ of cases, but King et al. [4] described 8 cases with obstruction of venous outflow in the transverse sinus, which disappeared after CSF extraction. They concluded that the likely cause of the obstruction was a collapse of the venous walls due to raised intracranial pressure, suggesting that stenosis was a secondary phenomenon. We would like to emphasize the importance of MR venography or CT angiography in IIH. Stent placement is often associated with a reduction in the pressure gradient and with clinical improvements. Such placement could preserve visual function, despite the difficulty of determining whether stenosis is a primary or a secondary event.

\section{References}

- 1 Skau M, Brennum J, Gjerris F, Jensen R: What is new about idiopathic intracranial hypertension? An updated review of mechanism and treatment. Cephalagia 2006;26: 384-399.

-2 Ball AK, Clarke CE: Idiopathic intracranial hypertension. Lancet Neurol 2006;5:433442 .

3 Higgins JN, Cousins C, Owler BK, Sarkies N, Pickard JD: Idiopathic intracranial hypertension: 12 cases treated by venous sinus stenting. J Neurol Neurosurg Psychiatry 2003;74:1662-1666.

4 King JO, Mitchell PJ, Thomson KR, Tress BM: Manometry combined with cervical puncture in idiopathic intracranial hypertension. Neurology 2002;58:26-30. 